\title{
Discovery and photometric study of the new in-the-gap SU UMa Dwarf Nova Var 73 Dra
}

\author{
S. V. Antipin ${ }^{1}$ and E. P. Pavlenko ${ }^{2}$ \\ ${ }^{1}$ Sternberg Astronomical Institute (SAI), 13, University Ave., Moscow 119992, Russia and Isaac Newton Institute of Chile, \\ Moscow Branch, Russia \\ e-mail: antipin@sai.msu.ru \\ 2 Crimean Astrophysical Observatory, Nauchny, 98409 Crimea, Ukraine and Isaac Newton Institute of Chile, Crimean Branch, \\ Ukraine \\ e-mail: pavlenko@crao.crimea.ua
}

Received 19 April 2002 / Accepted 6 May 2002

\begin{abstract}
We present the discovery and the first investigation of the new dwarf nova Var 73 Dra. We carried out monitoring and time-resolved CCD observations in 2001 August-October and detected several normal outbursts with a recurrence time of about 7-8 days and one slightly brighter superoutburst with a "plateau" phase that lasted 13 days. Our photometry during the superoutburst clearly shows the presence of superhumps with the period $0.0954( \pm 0.0001)$ day (although the one-day alias period, $0.1053 \mathrm{~d}$, could not be ruled out). So, the observed features allow us to classify Var 73 Dra as a new SU UMa-type dwarf nova within the well-known gap of orbital period distribution of cataclysmic variables.
\end{abstract}

Key words. stars: dwarf novae - stars: individual: Var73 Dra

\section{Introduction}

The modern theory of cataclysmic binaries explains the existence of the observed gap in the orbital-period distribution of dwarf novae conditioned by evolution of secondary stars (for review, see Osaki 1996). But recent papers show a presence of UGSU-type stars within the gap. And now we do not consider this feature of distribution as an absolute gap, but rather as a strong dearth of dwarf novae with orbital periods between 2.1 and 2.8 hours, especially at the upper part of the interval.

There are suggestions that the width and location of the period gap depend on characteristics of a secondary star of binary, as an example on its metallicity, inner structure, or magnetic field (Stehle et al. 1997; Hameury 1991). Thus, detailed research of dwarf novae in the gap may supply us with a very useful information about evolution of cataclysmic variables and about the peculiarity of systems with mass transfer from a companion star to a white dwarf persisting even within the mentioned interval of orbital periods.

In this paper we present a new discovery and first investigation of one more SU UMa-type star at the lower edge of the period gap.

Send offprint requests to: E. P. Pavlenko, e-mail: pavlenko@crao.crimea.ua

\section{Discovery and photographic observations}

The variability of Var 73 Dra was discovered by one of the authors (S. Antipin) on the Moscow archive plates taken with the 40-cm astrograph in Crimea. The star was identified with an object of the USNO-A2.0 catalogue (Monet et al. 1998) at $\alpha=20^{\mathrm{h}} 23^{\mathrm{m}} 38^{\mathrm{s}} 193, \delta=64^{\circ} 36^{\prime} 26^{\prime \prime} \cdot 91$ (J2000.0). The finding chart of Var 73 Dra is presented in Fig. 1.

The new variable was estimated by eye on 81 plates of the Moscow collection taken in JD2447730-49252 (1989-1993). The blue magnitudes of neighboring stars from the USNO-A2.0 catalogue were used for comparison.

The very limited number of plates did not allow us to analyse the brightness variations of the new variable in detail. But several general features have been revealed. Firstly, the new variable is fairly blue, with blue minus red color index -0.4 in the USNO-A2.0 catalogue. Secondly, the star clearly shows the outburst-type variability. These two characteristics let us consider Var 73 Dra as a new UG-type variable.

At least 14 outbursts of this dwarf nova have been observed photographically on the Moscow plates. The outbursts are relatively frequent. The $B_{\mathrm{pg}}$ magnitude of Var 73 reaches $15 \mathrm{~m} .8$ in the maximum brightness, and the star is not visible on our plates in the minimum (the limit of the best plates is $17 \cdot 6$ ). Unfortunately, no outburst has been observed well enough to reveal its shape or duration. The best-observed fragment of the photographic light curve is given in Fig. 2. The star seems to 


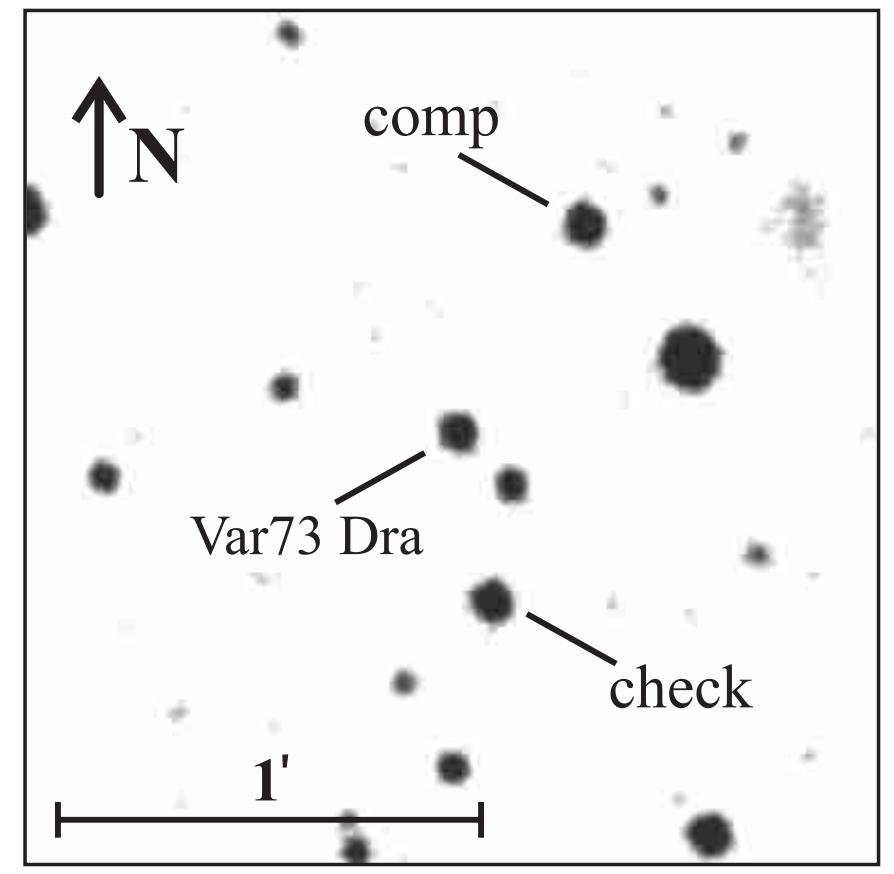

Fig. 1. The finding chart of Var 73 Dra based on POSS I red image, epoch 1953.6085. The variable star (at $\alpha=20^{\mathrm{h}} 23^{\mathrm{m}} 38^{\mathrm{s}} .193, \delta=$ $64^{\circ} 36^{\prime} 26^{\prime \prime} 91$ (J2000.0)) is very close to its maximum brightness in red light.

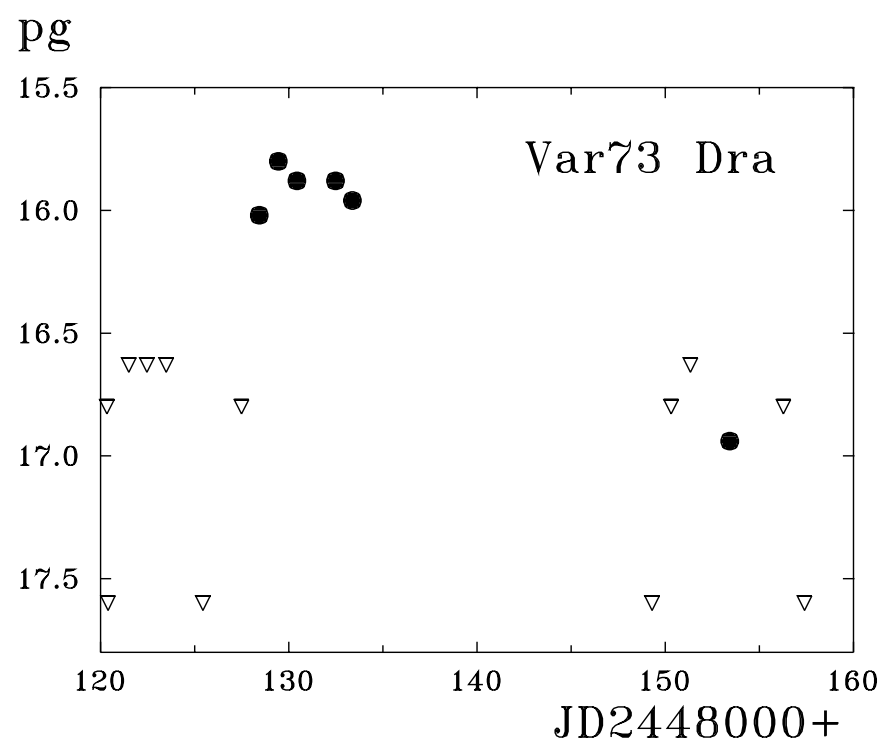

Fig. 2. Photographic light curve of Var 73 Dra in 1990 AugustSeptember. The positive observations are shown by filled circles. Open triangles give the limiting magnitude of plates with negative observations.

show two types of outbursts: the short-lasting ones (the brightening in JD 2448 153) and the longer ones (JD 2448 128-133).

To confirm the revealed outburst characteristics and to classify Var 73 Dra more definitely, we undertook CCD observations of this variable immediately after the discovery.

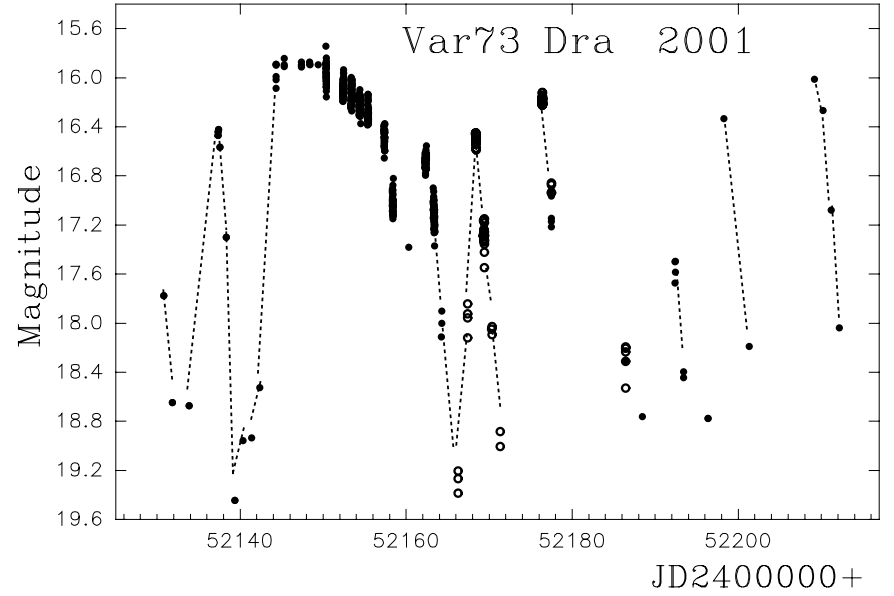

Fig. 3. The light curve of Var 73 Dra in 2001. The $R$ band observations are marked by filled circles, the data obtained in unfiltered light are shown as open circles.

\section{CCD observations}

CCD photometry was carried out in 2001 using a SBIG ST7 CCD camera and the $60-\mathrm{cm}$ reflector at the Crimean Laboratory of the Sternberg Astronomical Institute (SAI). Observations were made mostly in Johnson $R$ band, but sometimes in unfiltered light.

The images were dark subtracted, flat-fielded and analyzed with the profile/aperture photometry package developed by V.P. Goranskij. A total of 659 brightness measurements of Var 73 Dra were obtained for 44 nights in August-October, 2001. The log of observations is presented in Table 1. The unfiltered photometry is marked by "un" on the last column of the table.

We used the star from the USNO-A2.0 catalogue at $\alpha=$ $20^{\mathrm{h}} 23^{\mathrm{m}} 35^{\mathrm{s}} .358, \delta=64^{\circ} 36^{\prime} 56^{\prime \prime}$.66 (J2000.0) for comparison. Its $V$ and $R$ magnitudes were measured relative to the standard near V1504 Cyg (Pavlenko \& Dudka 2002) as the following: $V=16.33, R=15.58$. The USNO-A2.0 star at $\alpha=20^{\mathrm{h}} 23^{\mathrm{m}} 37^{\mathrm{s}} .450, \delta=64^{\circ} 36^{\prime} 02^{\prime \prime} .46(\mathrm{~J} 2000.0)$ was used as a check star. The check-comp difference in $R$ band was constant to within $0.01-0.02$. Both the comparison and the check stars are marked in Fig. 1. The typical accuracy of our CCD photometry was $0.01-0.02$ during the bright state of Var 73 Dra and about $0.1-0.2$ close to the minimum brightness of the star.

\section{CCD light curve and data analysis}

The CCD light curve of Var 73 Dra (Fig. 3) shows several short and one long-lasting outbursts. The shape of both short and long outbursts is typical of SU UMa-subtype stars. The longer and brighter outburst with a slow decline is similar to superoutbursts and more frequent brightenings resemble normal outbursts. The star is as faint as $R \sim 19$. 3 at minimum, and at maximum it reaches $R=15.9$, so the amplitude of the superoutburst is more than 3 mag. The "plateau" phase of the superoutburst has lasted about 13 days. The decline rate during this stage was $0.06 \mathrm{mag} /$ day. 
Table 1. Log of CCD observations.

\begin{tabular}{lccc|cccc}
\hline \hline $\begin{array}{l}\text { HJD } \\
2452000+\end{array}$ & $\begin{array}{c}\text { Exposure } \\
\text { time }(\mathrm{s})\end{array}$ & $\begin{array}{l}\text { Number } \\
\text { of exp. }\end{array}$ & Filter & $\begin{array}{l}\text { HJD } \\
2452000+\end{array}$ & $\begin{array}{c}\text { Exposure } \\
\text { time (s) }\end{array}$ & $\begin{array}{c}\text { Number } \\
\text { of }\end{array}$ & Filter \\
\hline 130.790 & 180 & 1 & $R$ & $163.263-.445$ & 180 & 72 & $R$ \\
131.858 & 180 & 1 & $R$ & $164.260-.273$ & 180 & 3 & $R$ \\
133.844 & 180 & 1 & $R$ & $166.249-.433$ & 360 & 7 & un \\
$137.376-.551$ & 180 & 4 & $R$ & $167.420-.433$ & 360 & 4 & un \\
138.330 & 180 & 1 & $R$ & $168.358-.447$ & 360 & 20 & un \\
139.380 & 180 & 1 & $R$ & $169.338-.440$ & 360 & 21 & un \\
140.363 & 180 & 1 & $R$ & $170.327-.354$ & 380 & 3 & un \\
141.364 & 180 & 1 & $R$ & $171.332-.347$ & 380 & 2 & un \\
142.378 & 180 & 1 & $R$ & $176.327-.423$ & 120 & 40 & un \\
$144.319-.332$ & 180 & 5 & $R$ & $177.467-.543$ & 180 & 4 & $R$ \\
$145.325-.330$ & 180 & 3 & $R$ & $177.476-.478$ & 120 & 2 & un \\
$147.369-.376$ & 180 & 4 & $R$ & $186.409-.414$ & 180 & 3 & un \\
$148.390-.398$ & 180 & 3 & $R$ & 186.432 & 360 & 1 & $R$ \\
149.405 & 180 & 1 & $R$ & 188.449 & 90 & 1 & $R$ \\
$150.341-.412$ & 120 & 33 & $R$ & $192.409-.414$ & 120 & 4 & $R$ \\
$152.347-.504$ & 180 & 57 & $R$ & $193.405-.409$ & 240 & 2 & $R$ \\
$153.344-.505$ & 180 & 54 & $R$ & 196.381 & 180 & 1 & $R$ \\
$154.312-.500$ & 180 & 84 & $R$ & 198.275 & 180 & 1 & $R$ \\
$155.334-.452$ & 180 & 46 & $R$ & 201.310 & 180 & 1 & $R$ \\
$157.306-.420$ & 180 & 38 & $R$ & 209.193 & 60 & 1 & $R$ \\
$158.328-.439$ & 180 & 46 & $R$ & 210.210 & 120 & 1 & $R$ \\
160.293 & 180 & 1 & $R$ & 211.200 & 240 & 1 & $R$ \\
$162.256-.452$ & 180 & 76 & $R$ & 212.181 & 200 & 1 & $R$ \\
\hline
\end{tabular}
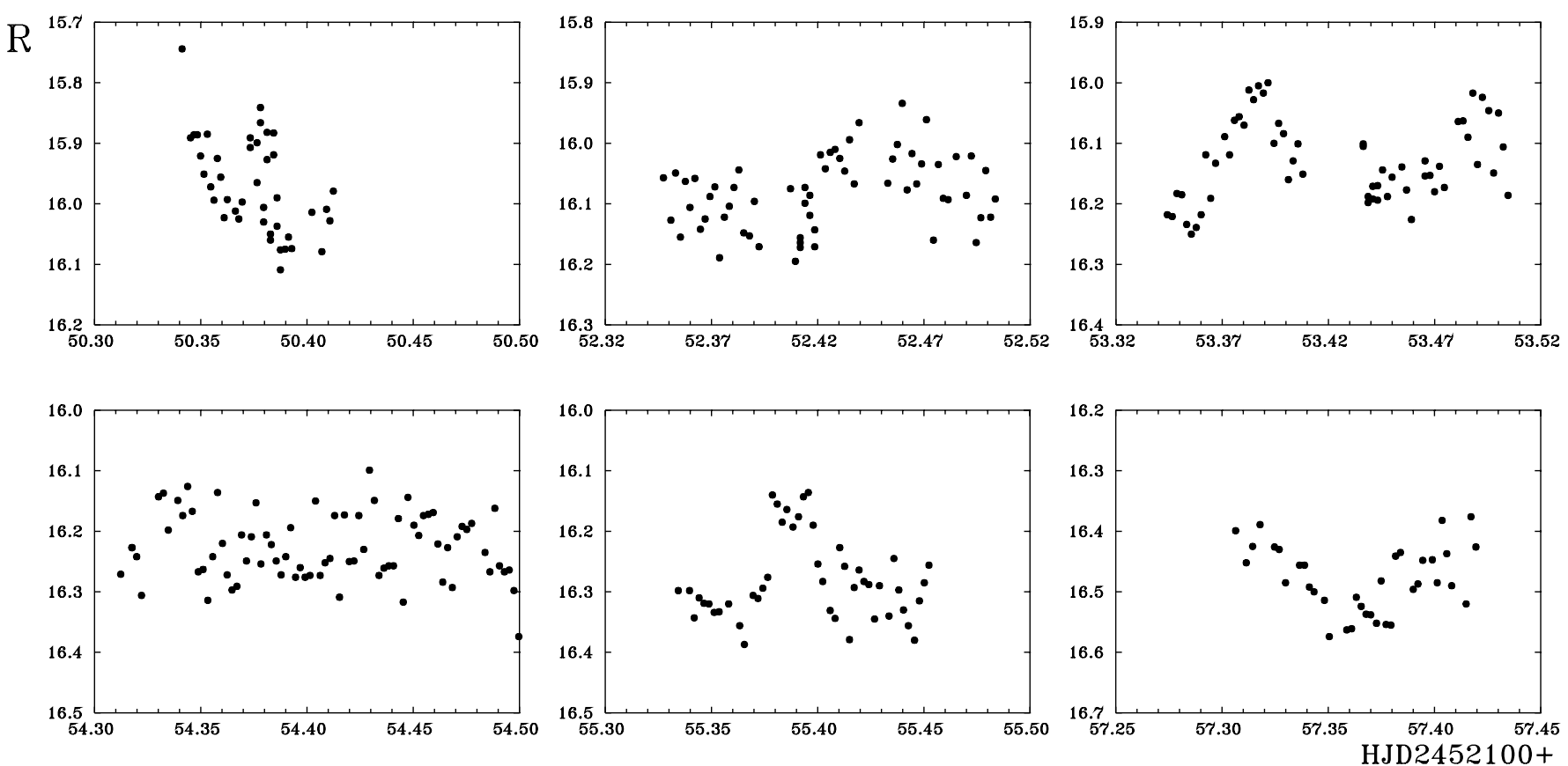

Fig. 4. Nightly light curves of Var 73 Dra during the superoutburst.

We present the results of our time-resolved CCD photometry during the superoutburst in Fig. 4. The light variations with the amplitude $0 .{ }^{\mathrm{m}} 1-0 \mathrm{~m} 2$, similar in shape and duration to superhumps of UGSU-type stars, are clearly seen, maybe with the exception of the date JD 2452 154. Immediately after the end of the superoutburst, the so-called "echo-outburst" or "rebrightening" was observed. Figure 3 shows that it lasted $\sim 6$ days (i.e. slightly longer then the normal outburst) and reached at least 16.4 in $R$ band. Close to the top of the rebrightening, we detected brightness variations on the time scale comparable to the length of the superhumps (see Fig. 5).

We analysed all data that shows hump-like features (including the top of the echo-outburst, but excluding the data obtained on JD 2452 154). After subtracting the trend that corresponded 


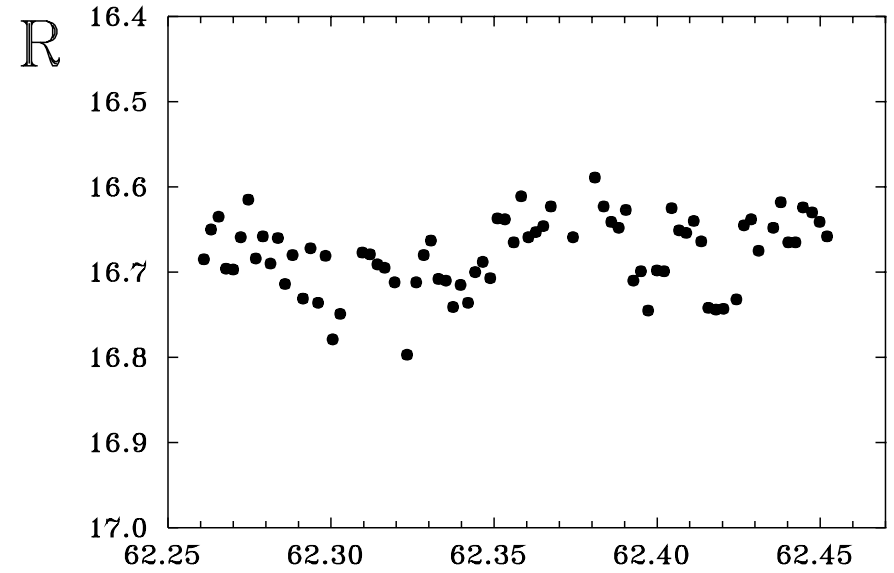

Fig. 5. The light curves of Var 73 Dra during the rebrightening.

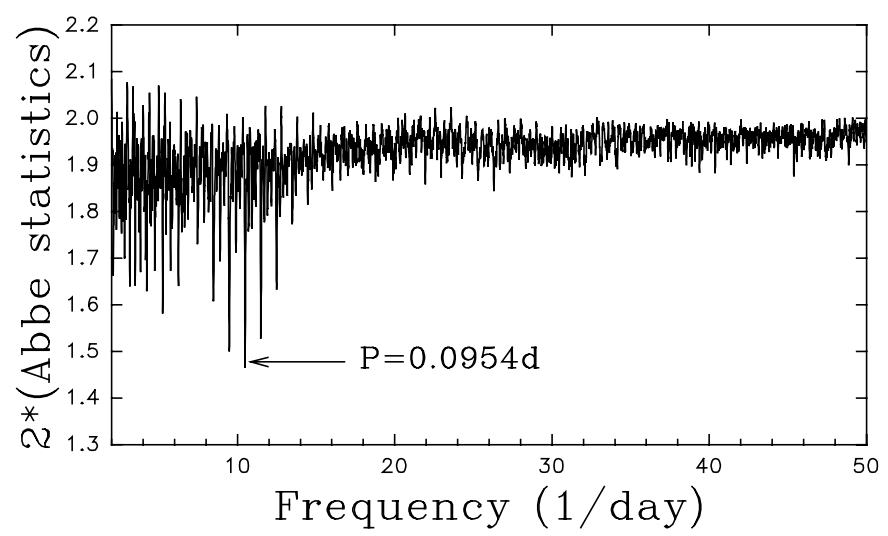

Fig. 6. The periodogram constructed using Stellingwerf's method. The strongest peak corresponds to the superhump period $0.0954 \pm 0.0001 \mathrm{~d}$.

to the mean brightness decline, we constructed a periodogram using Stellingwerf's method (Pelt 1980; Pelt 1992) with the Abbe statistics (Lafler \& Kinman 1965). The result is given in Fig. 6. There are three most significant peaks at 1-day aliased frequencies. The strongest peak conforms to the superhump period $P=0.0954 \pm 0.0001 \mathrm{~d}$.

The neighboring peak corresponds to period $0.105 \mathrm{~d}$ but it has less significance, so we preferred to consider $P=0.0954 \mathrm{~d}$ as the real period of superhumps. Note that both mentioned periods are located within the well-known gap of the orbital period distribution of cataclysmic variables. Our observations whitened for decline and folded with the superhump period are presented in Fig. 7. The mean profile has an asymmetric shape with steep increase, moderate decline, and amplitude of about 0 . 1 . The times of superhump maxima and minima are given in Table 2. Insufficient number of extrema did not allow us to examine changes of superhump period during the superoutburst.

Using the well-known empirical correlation between $\epsilon=$ $\left(P_{\text {sh }}-P_{\text {orb }}\right) / P_{\text {orb }}$ and $P_{\text {orb }}$ (Molnar \& Kobulnicky 1992; Patterson 2001), where $P_{\text {orb }}$ and $P_{\text {sh }}$ are the orbital and superhump periods respectively, we tried to estimate the value of orbital period. In the vicinity of the period gap, $\epsilon \sim 0.05$. So the best candidate orbital period could be $0.091 \mathrm{~d}$ (or $0.099 \mathrm{~d}$ for the less probable period). Even if we assume the largest
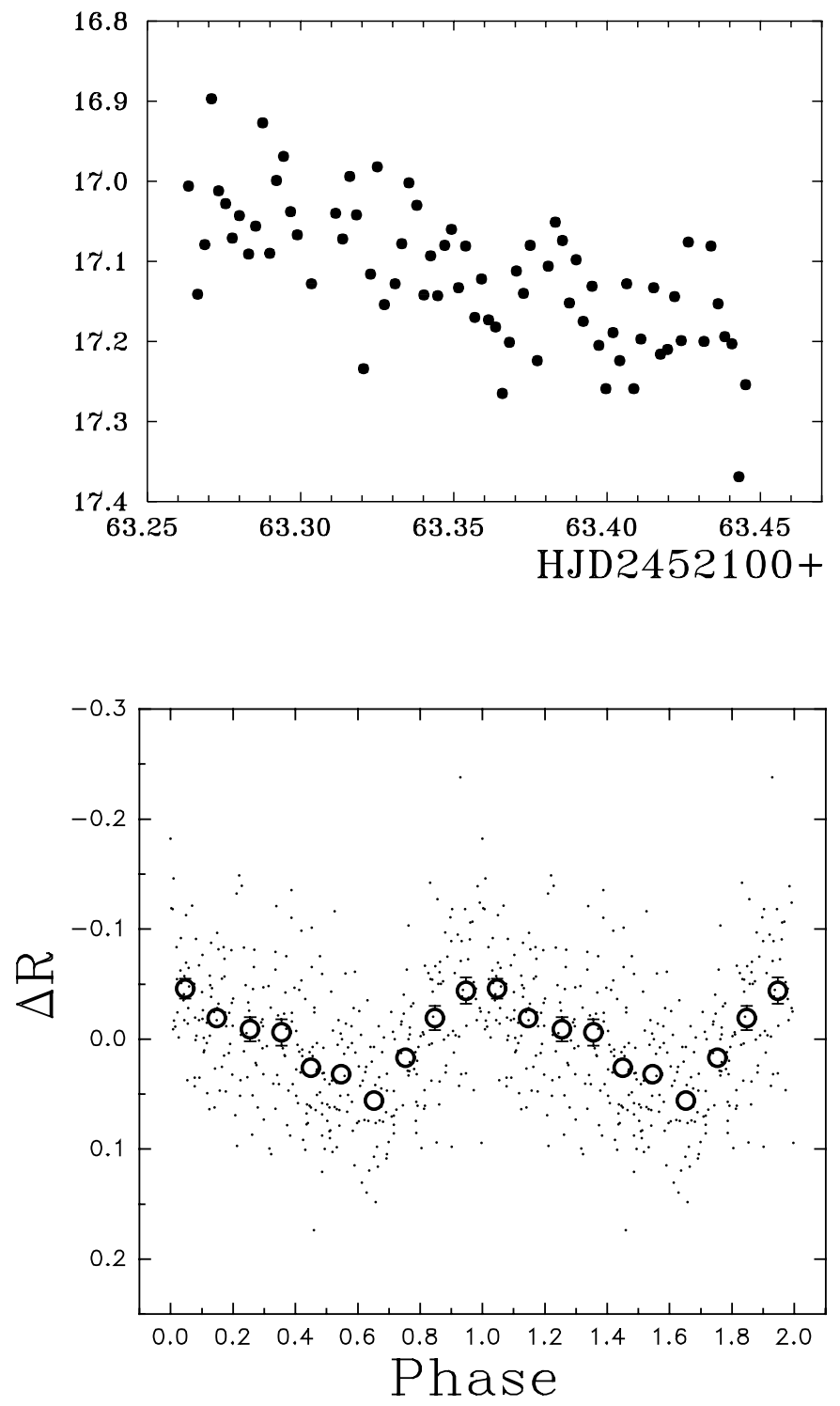

Fig. 7. The data folded on the superhump period $0.0954 \mathrm{~d}$. The mean superhump profile is given by open circles.

Table 2. Superhump extrema.

\begin{tabular}{cc}
\hline \hline $\begin{array}{c}\text { (HJD 2 452 000+) } \\
\text { Maxima }\end{array}$ & $\begin{array}{c}\text { (HJD 2 452 000+) } \\
\text { Minima }\end{array}$ \\
\hline $152.441 \pm 0.006$ & $150.398 \pm 0.14$ \\
$153.386 \pm 0.002$ & $152.397 \pm 0.11$ \\
$155.381 \pm 0.003$ & $153.455 \pm 0.008$ \\
$162.373 \pm 0.006$ & $157.355 \pm 0.006$ \\
& $162.315 \pm 0.006$ \\
& $162.419 \pm 0.009$ \\
\hline
\end{tabular}

superhump excess known for SU UMa-type stars $(7.17 \%$ for TU Men), the orbital period corresponding to $P_{\mathrm{sh}}=0.0954$ will be 2.13 hours, i.e. it will be disposed within the gap. 


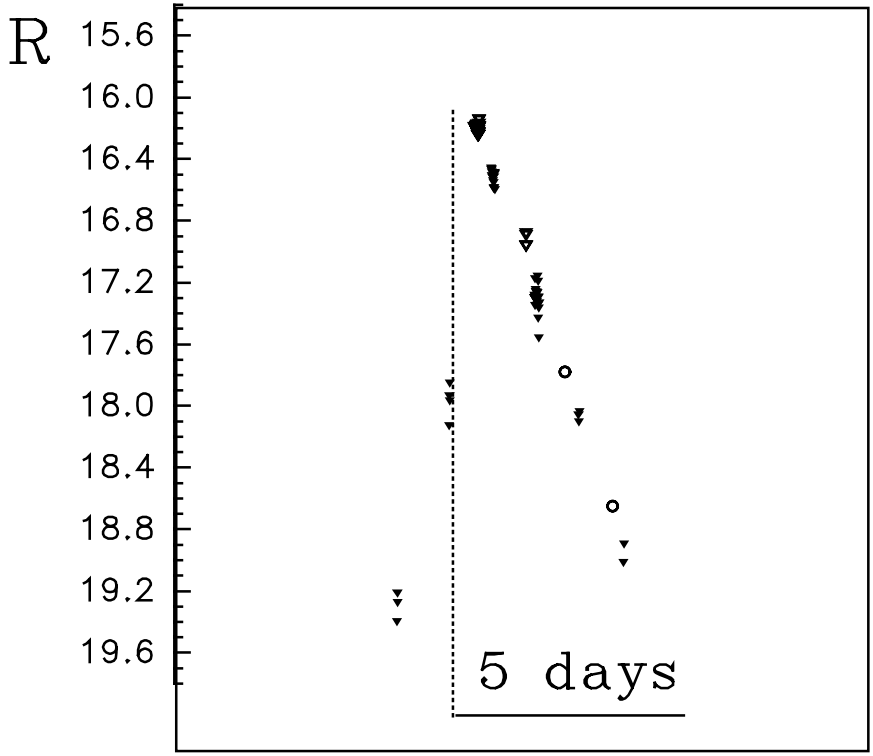

Time

Fig. 8. The summary profile of the normal outburst. The original outbursts are marked by different symbols. The dotted line is drawn through the maximum.

\section{Normal outbursts}

Visual inspection of the CCD light curve yields the typical frequency of normal outbursts of 7-8 days. To determine the outburst's shape, amplitude and duration, we constructed the composite normal outburst by moving the original observed profiles along the time axis until the best fit was obtained. The light curve of this "summary" outburst is presented in Fig. 8. It shows a very sharp ascending branch and moderate decline that lasted $\sim 3$ days. This shape is typical for the so-called "type A" outbursts (Smak 1984), which can be explained in the disk instability model as a result of outside-in propagation of the heating front in the accretion disk (the thermal instability occurs in the outer part of the disk and then propagates inside).

\section{Conclusions}

We can summarize our results as follows:

1. The new UG-type variable was discovered; already its photographic photometry permitted us to consider this star a candidate to UGSU-subtype dwarf novae.

2. CCD photometry of Var 73 Dra in August-October 2001 clearly showed the existence of two types of outbursts strongly differing in length: short normal outbursts and a long superoutburst.

3. The normal outbursts are relatively frequent, their duration is close to 5 days, the recurrence time is about 7-8 days.

4. The observed superoutburst is slightly brighter and appreciably longer then the normal ones.

5. The analysis of the time-resolved CCD photometry during the plateau phase of the superoutburst resulted in the discovery of superhumps with period $\left(P_{\mathrm{sh}}=0.0954\right)$ located within the well-known period gap, close to its lower edge.
Table 3. Known SU UMa stars in the gap.

\begin{tabular}{lll}
\hline \hline Star & $P_{\text {sh }}$ (days) & Source of $P_{\text {sh }}$ \\
\hline GX Cas & $0.09297 \pm 0.00005$ & Nogami et al. (1998b) \\
V419 Lyr & 0.0923 or 0.0953 & Nogami et al. (1998b) \\
Var73 Dra & $0.0954 \pm 0.0001$ & this Paper \\
V725 Aql & $0.09909 \pm 0.00015$ & Uemura et al. (2001) \\
NY Ser & $0.106 \pm 0.001$ & Nogami et al. (1998a) \\
TU Men & 0.1256 (variable) & Stolz \& Schoembs (1984) \\
\hline
\end{tabular}

6. The so-called rebrightening was observed after the end of the superoutburst.

All revealed characteristics unequivocally show that Var 73 Dra is a new in-the-gap UGSU-type binary.

We would like to note that the lower part of the gap is more and more populated with known SU UMa-type dwarf novae. In fact, the gap becomes narrower, the lower border of strong deficiency moves towards longer periods. To illustrate our statement, we display in Table 3 the data on superhump periods of six well-investigated UGSU stars in the gap. Here we accept the lower border of the gap at $P_{\mathrm{sh}} \sim 2.2$ hours $(0.0917 \mathrm{~d})$, corresponding to $P_{\text {orb }} \sim 2.1$ hours. For V419 Lyr, the table presents two more probable of the four possible values of the superhump period from Nogami et al. (1998b).

Acknowledgements. The authors would like to thank Dr. N. N. Samus for his help and useful discussion. S. Antipin is grateful to the Russian Foundation for Basic Research (grants Nos. 02-02-16462 and 02-02-06569) and the Council of the Program for the Support of Leading Scientific Schools (grant No. 00-15-96627) for partial support of this research. E. Pavlenko acknowledges partial support from Ukrainian Foundation for Fundamental Research through the grant 02/07/00451.

\section{References}

Hameury, J. M. 1991, A\&A, 243, 419

Lafler, J., \& Kinman, T. D. 1965, ApJS, 11, 216

Molnar, L. A., \& Kobulnicky, H. A. 1992, ApJ, 392, 678

Monet, D., et al. 1998, USNO-A2.0, A Catalog of Astrometric Standards (U.S. Naval Observatory, Washington, DC)

Nogami, D., Kato, T., Baba, H., \& Masuda, S. 1998a, PASJ, 50, L1

Nogami, D., Kato, T., \& Masuda, S. 1998b, PASJ, 50, 411

Osaki, Y. 1996, PASP, 108, 39

Patterson, J. 2001, PASP, 113, 736

Pavlenko, E. P., \& Dudka, O. I. 2002, Astrofizika, 45, 5

Pelt, J. 1980, Frequency analysis of astronomical time series (Valgus publ., Tallinn)

Pelt, J. 1992, Irregulary Spaced Data Analysis, User manual, Helsinki Smak, J. 1984, Acta Astron., 34, 161

Stehle, R., Kolb, U., \& Ritter, H. 1997, A\&A, 320, 136

Stolz, B., \& Schoembs, R. 1984, A\&A, 132, 187

Uemura, M., Kato, T., Pavlenko, E., Baklanov, A., \& Pietz, J. 2001, PASJ, 53, 539 